\title{
On parallel displacements in Finsler spaces.
}

\author{
By Masao Hashiguchi
}

(Received Dec. 26, 1957)

The notion of parallelism was introduced in Finsler spaces by many authors, who gave interesting definitions thereof from various points of view. Most of these parallelisms are derived from some systems of axioms, but the formulas representing them are more complicated than in the case of Riemannian geometry. So it seems to be interesting that we return to the foundation of the theory and consider whether the complications of the formulas are essentially unavoidable or not.

In this note we shall investigate what conditions are to be imposed on connections in Finsler spaces in order that the analogous properties still hold in our spaces as in Riemannian geometry. As the results of this consideration we shall get a characterization of a Finsler metric among more general metrics (Theorem 3) and it will be shown that the connection defined by E. Cartan [4] as stated in Theorem 1] is the shortest and the fittest from our standpoint (Theorem 2 and $13^{\circ}$ ). Further, we can immediately derive from it the parallelisms defined by other authors. The parallel displacements defined by J.L. Synge [1], J. H. Taylor [2] and W. Barthel [7] are special cases of the one by E. Cartan, and the connections defined by H. Rund [5], [6] and L. Berwald [3] are immediately obtained from it by applying Lemma 2 and Lemma 3 respectively; and these lemmas give us two methods to define new connections from a given one (Theorem.4).

I wish to express my sincere gratitude to Prof. M. Matsumoto for the invaluable suggestions and critisisms during the preparation of this note.

\section{$\S 1$. Spaces with line-elements.}

$1^{\circ}$. When we deal with the geometry of the differentiable manifold $\widetilde{F}$ such that its properties depend not only on the points of $\mathfrak{F}$ but also on the non-zero tangent vectors at each point, we shall call $\mathfrak{F}$ the space with lineelements and the tangent vector the supporting element.

The set of all non-zero tangent vectors at the point $p$ is called the tangent space at $p$ and denoted by $T_{p}$. The set $\mathfrak{B}(\mathfrak{\wp})$ of all non-zero tangent vectors on $\widetilde{F}$ is considered as a differentiable manifold, which is called the tangent bundle. As is well known, the tangent vector $X$ at the point $p$ is locally 
expressed by $X=X^{i} \partial / \partial x^{i}$ in terms of the local coordinates $x^{i}$ defined in the neighborhood of $p$ and the set $\left(x^{1}, \cdots, x^{n}, X^{1}, \cdots, X^{n}\right)$ is used as the local coordinates of $\mathfrak{B}(\mathfrak{F})$. When a tangent vector $X$ is regarded as a supporting element, we shall specifically write $Y$ and $\left(x^{i}, y^{i}\right)$ instead of $X$ and $\left(x^{i}, X^{i}\right)$ respectively.

$2^{\circ}$. When the regular curve $\widetilde{C}$ in $\mathfrak{B}(\widetilde{\mho})$ is mapped on the regular curve $C$ in $\widetilde{\mho}$ by the projection to the base space $\widetilde{\mho}$, this curve $\widetilde{C}$ is called the regularly covering curve of $C$. For example, if we have a regular curve $C$ in $\mathfrak{F}$ expressed locally by $x^{i}=x^{i}(t)$, then a curve $\tilde{C}$ expressed locally by $x^{i}=x^{i}(t)$, $y^{i}=a(t) d x^{i} / d t$ is a regular curve in $\mathfrak{B}(\mathfrak{F})$ and covers $C$ regularly, where $a(t)$ is to be everywhere positive and differentiable. We shall denote this curve in $\mathfrak{B}(\mathfrak{F})$ by $\widetilde{C}_{a}$, which does not of course depend on a special coordinate system in $\widetilde{F}$. And when a differentiable vector field $Y$ is given, we can take a regularly covering curve $\tilde{C}_{Y}$ for any regular curve $C$ in $\mathfrak{F}$ by the natural way as follows. That is, expressing locally $C$ and $Y$ by $x^{i}=x^{i}(t)$ and $Y=y^{i} \partial / \partial x^{i}$ respectively, then $\widetilde{C}_{Y}$ is defined by $x^{i}=x^{i}(t), y^{i}=y^{i}(x(t))$.

$3^{\circ}$. When we deal with the function defined on $\mathfrak{B}(\mathfrak{F})$, we have to pay attention to its homogeneity and use the well-known Euler's relation if we need. We shall first give a few words about them. Let $f$ be a function defined on $\mathfrak{B}(\mathfrak{F})$. If, at every point $X$ of $\mathfrak{B}(\mathfrak{F})$, the relation $f(\lambda X)=\lambda^{m} f(X)$ is satisfied for any $\lambda>0$ and a fixed integer $m$, then this function $f$ is called the positively homogeneous function of degree $m$. In terms of local coordinates $\left(x^{i}, y^{i}\right)$ of $\mathfrak{B}(\mathfrak{F})$ this relation is expressed by $f(x, \lambda y)=\lambda^{m} f(x, y)$. In this case the following relations are known, that is:

$$
\frac{\partial f}{\partial y^{i}} y^{i}=m f, \quad \frac{\partial^{2} f}{\partial y^{i} \partial y^{j}} y^{j}=(m-1) \frac{\partial f}{\partial y^{i}} \quad \text { etc. }
$$

and $\partial f / \partial y^{i}$ is positively homogeneous of degree $m-1$.

$4^{\circ}$. Let $T_{p}$ be a tangent space at $p$ in $\widetilde{F}$ and $D_{p}$ its dual space. A tensor of type $(s, r), s$ being contravariant order and $r$ covariant order, is defined to be a mapping of the direct product

$$
T_{p} \underbrace{\times \cdots \cdots \times T_{p}}_{r \text {-times }} \times D_{p} \underbrace{\times \cdots \cdots \times D_{p}}_{s \text {-times }} \times T_{p}
$$

into $R$, assumed to satisfy the conditions:

1) it is multilinear in the first $r+s$ spaces,

2) it is positively homogeneous of degree zero in the last space.

The rule $g$ which assigns to every point $p \in \mathfrak{F}$ a tensor $g_{p}$ of type $(s, r)$ is called a tensor field of type $(s, r)$ on $\widetilde{\xi}$. To any differentiable vector fields $X_{1}, \cdots, X_{r}$ on $\widetilde{F}$ and any differentiable 1 -forms $\omega_{1}, \cdots, \omega_{s}$, corresponds the function $\tilde{g}\left[X_{1}, \cdots, X_{r}, \omega_{1}, \cdots, \omega_{s}\right]$ by the relation

$$
\tilde{g}\left[X_{1}, \cdots, X_{r}, \omega_{1}, \cdots, \omega_{s}\right]\left(Y_{p}\right)=g_{p}\left(\underset{p}{X_{1}}, \cdots, \underset{p}{X_{r}}, \underset{p}{\omega_{1}}, \cdots, \underset{p}{\omega_{s}}, Y_{p}\right)
$$


for all $Y_{p} \in \mathfrak{B}(\mathfrak{F})$. The tensor field $g$ is called differentiable if $\tilde{g}\left[X_{1}, \cdots, X_{r}\right.$, $\left.\omega_{1}, \cdots, \omega_{s}\right]$ is the differentiable function on $\mathfrak{B}(\mathfrak{F})$ for any differentiable $X_{i}$ and $\omega_{i}$. For a coordinate system $x^{i}$ we put

$$
g_{i_{1} \cdots i_{r}}^{j_{1} \cdots j_{s}}=\tilde{g}\left[\frac{\partial}{\partial x^{i_{1}}}, \cdots, \frac{\partial}{\partial x^{i_{r}}}, d x^{j_{1}}, \cdots, d x^{j_{s}}\right],
$$

which are called the components of $g$ in terms of this coordinate system. Let $g_{\alpha_{1} \cdots \alpha_{r}} \beta_{1 \cdots} \cdots \beta_{s}$ be the components of $g$ in terms of new local coordinates $x^{\alpha}$, then we have the following law of transformation:

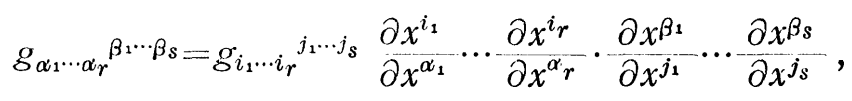

\section{$\S 2$. The notions of metric and connection.}

$5^{\circ}$. Now we introduce a metric in our space $\mathfrak{F}$. This is defined as a differentiable tensor field of type $(0,2)$ which satisfies the following two conditions:

1) $\tilde{g}[X, Y]=\tilde{g}[Y, X]$ for any differentiable vector fields $X$ and $Y$,

2) $\tilde{g}[X, X]>0$ for any non-zero differentiable vector field $X$.

Now we have $n^{2}$ differentiable functions $g_{i j}$ on $\mathfrak{B}(\mathfrak{F})$, which are components of the metric tensor $g$ in terms of local coordinates: $g_{i j}=\tilde{g}\left[\frac{\partial}{\partial x^{i}}, \frac{\partial}{\partial x^{j}}\right]$. By means of the above definitions of the tensor field and the metric, we can easily see that

1) $g_{i j}=g_{j i}$,

2) Let $x^{\alpha}$ be new local coordinates, and if $g_{\alpha \beta}=\tilde{g}\left[\frac{\partial}{\partial x^{\alpha}}, \frac{\partial}{\partial x^{\beta}}\right]$, then

$$
g_{\alpha \beta}=g_{i j} \frac{\partial x^{i}}{\partial x^{\alpha}} \frac{\partial x^{j}}{\partial x^{\beta}},
$$

3) $g_{i j}(x, y) t^{i} t^{j}$ is positive-definite for $t \in R$,

4) $g_{i j}(x, \lambda y)=g_{i j}(x, y)$ for any $\lambda>0$.

Conversely, if we have the system of functions $g_{i j}$ on $\mathfrak{B}(\mathfrak{F})$ satisfying the above four conditions, we may define a metric by these functions and then the $g_{i j}$ are components of the metric. We shall call $g$ the fundamental metric tensor. As usual we can get the $g^{i j}$ as the inverse of the $g_{i j}$, which determine a tensor of type $(2,0)$.

$6^{\circ}$. Let a metric $g$ be introduced in our space. To $X_{p} \in T_{p}$ we shall call

$$
\left|X_{p}\right|_{Y_{p}}=\sqrt{g_{p}\left(X_{p}, X_{p}, Y_{p}\right)}
$$

the length of $X_{p}$ with respect to the supporting element $Y_{p}$. In particular, we have the length of $X_{p}$ with respect to $X_{p}$ itself, which is called the absolute 
length of $X_{p}$. In terms of local coordinates we can express the length of the vector $X_{p}$ with respect to the supporting element $Y_{p}$ by the form

$$
\left|X_{p}\right|_{Y_{p}}=\sqrt{g_{i j}\left(x_{p}, y_{p}\right) X_{p}^{i}, X_{p}^{j}} .
$$

The arc-length of a regular curve $C$ with respect to the regularly covering curve $\tilde{C}$ is defined by

$$
s_{\tilde{C}}=\int_{t_{0}}^{t} \sqrt{g_{i j}(x, y) \frac{d x^{i}}{d t} \frac{d x^{j}}{d t}} d t,
$$

where $C$ and $\tilde{C}$ are expressed by the equations $x^{i}=x^{i}(t)$ and $x^{i}=x^{i}(t), y^{i}=y^{i}(t)$ respectively. In particular, the length with respect to $\widetilde{C}_{a}$ is called the absolute arc-length, where the curve $\widetilde{C}_{a}$ is the one defined in $2^{\circ}$. In this place we should note that, owing to the homogeneity of $g_{i j}$, an arc-length does not depend on the parameter of $C$, while the absolute length of the curve is not necessarily equal to the length of its anti-wised curve.

$7^{\circ}$. An affine connection is defined by a set of differentiable 1 -forms $\omega_{j}{ }^{i}$ on $\mathfrak{B}(\mathfrak{F})$ for each system of local coordinates in $\mathfrak{F}$, such that, when we set $\omega_{j}^{i}=\Gamma_{j k}^{i} d x^{k}+C_{j k}^{i} d y^{k}$,

1) these coefficients satisfy the following law of transformation:

$$
\begin{aligned}
& \Gamma_{\beta}{ }^{\alpha} r=\Gamma_{j k}{ }^{i} \frac{\partial x^{\alpha}}{\partial x^{i}} \frac{\partial x^{j}}{\partial x^{\beta}} \frac{\partial x^{k}}{\partial x r}+\frac{\partial x^{\alpha}}{\partial x^{i}} \frac{\partial^{2} x^{i}}{\partial x^{\beta} \partial x r} \\
& +C_{j k}^{i} \frac{\partial x^{\alpha}}{\partial x^{i}} \frac{\partial x^{j}}{\partial x^{\beta}} \frac{\partial^{2} x^{k}}{\partial x^{r} \partial x^{\delta}} \frac{\partial x^{\delta}}{\partial x^{l}} y^{l}, \\
& C_{\beta}{ }^{\alpha}{ }^{i}=C_{j k}{ }^{i}{ }_{k} \frac{\partial x^{\alpha} \partial x^{j} \partial x^{k}}{\partial x^{i} \partial x^{\beta} \partial x^{r}},
\end{aligned}
$$

2) the $\Gamma_{j k}^{i}$ are positively homogeneous of degree 0 ,

3) the $C_{j k}{ }^{i}$ are positively homogeneous of degree -1 and further

$$
C_{j k}{ }^{i} y^{k}=0 \text {. }
$$

It is easily seen that the definition is consistent, though we have defined in terms of local coordinates.

From now on we shall suppose that such an affine connection be given. Let $C$ be a regular curve and $\widetilde{C}$ be a regularly covering curve of $C$. Tangent vectors $X(t)$ are said to be parallel along the curve $C$ with respect to $\tilde{C}$ if the equations

$$
\frac{d X^{i}}{d t}+\Gamma_{j k}{ }^{i}(x, y) X^{j} \frac{d x^{k}}{d t}+C_{j k}{ }^{i}(x, y) X^{j} \frac{d y^{k}}{d t}=0
$$

are satisfied. When we take $\widetilde{C}_{a}$ as $\tilde{C}$, the parallelism is called to be absolute. In this case the vectors $X(t)$ satisfy the equations

$$
\frac{d X^{i}}{d t}+\Gamma_{j k}^{i}\left(x, \frac{d x}{d \iota^{i}}\right) X^{j} \frac{d x^{k}}{d t}+C_{j k}^{i}\left(x, \frac{d x}{d t}\right) X^{j} \frac{d^{2} x^{k}}{d t^{2}}=0
$$


for any differentiable $a(t)>0$. We shall notice that the last two conditions for the $\Gamma_{j k}^{i}$ and $C_{j k}^{i}$ are essential in order that the absolute parallelism does not depend on the parameter of $C$.

We can parameterize the curve $C$ by its absolute arc-length $s$, and then $C$ is called a relative geodesic with respect to $\tilde{C}$, if its tangent vector $d x^{i} / d s$ is parallel along $C$ with respect to $\tilde{C}$. And if it is absolutely parallel along $C$, then the curve $C$ is called the absolute geodesic, whose equations are as follows:

$$
\frac{d^{2} x^{i}}{d s^{2}}+\Gamma_{j k}{ }^{i}\left(x, \frac{d x}{d s}\right) \frac{d x^{j}}{d s} \frac{d x^{k}}{d s}+C_{j k}{ }^{i}\left(x, \frac{d x}{d s}\right) \frac{d x^{j}}{d s} \frac{d^{2} x^{k}}{d s^{2}}=0 .
$$

Now we should show that the parallelism thus defined does not depend on a special coordinate system in $\widetilde{F}$. This is shown from the following consideration. We sct for any vector field $X$ on $\mathfrak{F}$

$$
D X^{i}=d X^{i}+\omega_{j}^{i} X^{j}
$$

in terms of the local coordinates $x^{i}$. In order that the operation $D$ has the geometrical meaning, the following law of transformation should be satisfied:

$$
D X^{\alpha}=D X^{i} \frac{\partial x^{\alpha}}{\partial x^{i}},
$$

where $X^{\alpha}$ are components of $X$ in terms of new local coordinates $x^{\alpha}$. We shall find the conditions for this. We have

$$
\begin{aligned}
& D X^{\alpha}=d X^{\alpha}+\Gamma_{\beta}{ }^{\alpha}{ }_{r} X^{\beta} d x r+C_{\beta}{ }^{\alpha}{ } X^{\beta} d y r \\
& =D X^{i} \frac{\partial x^{\alpha}}{\partial x^{i}} \\
& +\left(\Gamma_{\beta}^{\alpha} r \frac{\partial x^{\beta}}{\partial x^{j}} \frac{\partial x r}{\partial x^{k}}-\Gamma_{j k}^{i} \frac{\partial x^{\alpha}}{\partial x^{i}}+\frac{\partial^{2} x^{\alpha}}{\partial x^{j}} \frac{\partial x^{k}}{\partial C_{\beta} r} \frac{\partial x^{\beta}}{\partial x^{j}} \frac{\partial^{2} x^{r}}{\partial x^{k} \partial x^{l}} y^{l}\right) X^{j} d x^{k} \\
& +\left(C_{\beta}{ }^{\alpha} r \frac{\partial x^{\beta}}{\partial x^{j}} \frac{\partial x r}{\partial x^{k}}-C_{j k}^{i} \frac{\partial x^{\alpha}}{\partial x^{i}}\right) X^{j} d y^{k},
\end{aligned}
$$

hence in order that the relation (6) holds for any $X$, it is necessary and sufficient that

$$
\begin{aligned}
& \Gamma_{\beta}^{{ }^{k}} r \frac{\partial x^{\beta}}{\partial x^{j}} \frac{\partial x r}{\partial x^{k}}-\Gamma_{j k}^{i} \frac{\partial x^{\alpha}}{\partial x^{i}}+\frac{\partial^{2} x^{\alpha}}{\partial x^{j} \partial x^{k}}+C_{\beta}{ }^{a} r \frac{\partial x^{\beta}}{\partial x^{j}} \frac{\partial^{2} x r}{\partial x^{k} \partial x^{l}} y^{l}=0, \\
& C_{\beta}{ }^{\alpha} r \frac{\partial x^{\beta}}{\partial x^{j}} \frac{\partial x r}{\partial x^{k}}-C_{j k}^{i} \frac{\partial x^{\alpha}}{\partial x^{i}}=0,
\end{aligned}
$$

and from these equations we have the equations (1) and (2) as the conditions to be found. This is the reason why we imposed the condition 1 ) on the coefficients of the connection.

$8^{\circ}$. When the length of a vector remains unchanged under the parallel 
displacement along a curve, the connection is called to be metrical. This condition is expressed as $d\left(g_{i j} X^{i} X^{j}\right)=0$ for any parallel vector field $X$, and from this we have

Lemma 1. (E. Cartan) The connection is metrical if and only if

where we put

$$
\begin{aligned}
& \frac{\partial g_{i j}}{\partial x^{k^{-}}}=\Gamma_{i j k}+\Gamma_{j i k}, \\
& \frac{\partial g_{i j}}{\partial y^{k}}=C_{i j k}+C_{j i k},
\end{aligned}
$$

$$
\Gamma_{i j k}=g_{j h} \Gamma_{i k}^{h} \text { and } C_{i j k}=g_{j h} C_{i k}^{h} \text {. }
$$

$9^{\circ}$. By means of a system of linear differential equations

$$
\left\{\begin{aligned}
\frac{d \boldsymbol{P}}{d t} & =\frac{d x^{i}}{d t} \boldsymbol{e}_{i} \\
d \boldsymbol{e}_{i} & =\left(\Gamma_{i{ }_{k}{ }^{j}} \frac{d x^{k}}{d t}+C_{i^{\prime}{ }_{k}} \frac{d y^{k}}{d t}\right) \boldsymbol{e}_{j} \quad(i=1,2, \cdots, n)
\end{aligned}\right.
$$

for $n+n^{2}$ unknown functions $\boldsymbol{P}$ and $\boldsymbol{e}_{i}$ of one variable $t$, any curve $C$ in $\widetilde{\mho}$ and the one-parametric tangent spaces along $C$ may be developed in an Euclidean space $E^{n}$ in the similar way as in Riemannian geometry, and then most properties in Riemannian geometry still hold in our space. For example, the developed vectors of any parallel vector field along a curve in $\mathfrak{F}$ are also parallel in $E^{n}$, and the geodesic in $\mathfrak{F}$ is developed upon a straight line in $E^{n}$. In case a metric is introduced in $\mathfrak{F}$, we take initial conditions in (9) such that $\boldsymbol{e}_{i} \boldsymbol{e}_{j}=g_{i j}$. Then, if the connection is metrical, the Euclidean length of the developed vector is equal to the one of the corresponding vector in $\mathfrak{F}$ and so on.

Now we suppose that coefficients $C_{j k}{ }^{i}$ of a connection satisfy the equations

$$
C_{j k}^{i} y^{j}=0 \text {. }
$$

Under the parallel displacement along a curve $C$, if we take in particular the supporting element $Y$ to be parallel, i. e.

$$
\frac{d y^{i}}{d t}+\Gamma_{j k}^{i} y^{j} \frac{d x^{k}}{d t}+C_{j k}{ }_{k} y^{j} \frac{d y^{k}}{d t}=0 \text {, }
$$

then we have

$$
\frac{d y^{i}}{d t}=-\Gamma_{j k}^{i} y^{j} \frac{d x^{k}}{d t}
$$

and consequently the equations $(9)_{2}$ may be written in the form

where

$$
\frac{d \boldsymbol{e}_{i}}{d t}=\Gamma_{i k}^{* j} \frac{d x^{k}}{d t} \boldsymbol{e}_{j}
$$




$$
\Gamma_{j k l}^{* i}=\Gamma_{j k}^{i}-C_{j l}^{i} \Gamma_{m k}^{l} y^{m} .
$$

If we develope an infinitesimal circuit $(d x, \delta x)$ in an Euclidean space $E^{n}$, then it is easily seen that the difference between the initial point and the terminal one is expressed by $\left(\Gamma_{j k}^{* i}-\Gamma_{k j}^{* i}\right) \times \delta x^{j} d x^{k} \boldsymbol{e}_{i}$. Since [12) has a solution, we can always take the regularly covering curve $\tilde{C}$ such that its $y^{i}$-coordinates satisfy (12). However by the direct calculation independently of the above geometrical consideration, we observe that the quantities

$$
S_{j k}^{i}=\Gamma_{j k}^{* i}-\Gamma_{k j}^{* i}
$$

are the components of a tensor field of type $(1,2)$, where the condition (10) is essential. This tensor field depends only on the affine connection and is called the torsion tensor field of this connection.

Moreover, in the above calculation we notice that the $\Gamma_{j k i}^{* i}$ and $C_{j k}^{* i}(=0)$ satisfy all of the conditions for the coefficients $\Gamma_{j k}{ }^{i}$ and $C_{j k}{ }^{i}$ of the connection. Hence we have

Lemma 2. Let a connection be defined in the space $\mathfrak{F}$ by the quantities $\Gamma_{j}{ }^{t}$ k and $C_{j k}{ }^{i}$ such that $C_{j k}{ }^{i} y^{j}=0$. Then we can define a new connection in $\mathfrak{F}$ by the above $\Gamma_{j k}^{* i}$ and $C_{j k}^{* i}$. This is not in general metrical unless the supporting element is taken to be parallel, even if the original connection is metrical.

Finally we remark that the quantities

$$
\tilde{\Gamma}_{j k}^{i}=\frac{1}{2} \frac{\partial^{2}}{\partial y^{j} \partial y^{t_{i}}}\left(\Gamma_{h}{ }^{i}{ }_{l} y^{h} y^{l}\right)
$$

and $C_{j k}^{* i}(=0)$ satisfy all of the conditions for coefficients $\Gamma_{j k}{ }^{i}$ and $C_{j k}{ }^{i}$ of the connection. This follows immediately from the equation (1), making use of (10)! Thus we have

Lemma 3. Under the same assumption as in Lemma 2 we can define a new connection in $\Im$ by the above $\tilde{\Gamma}_{j k}{ }^{i}$ and $C_{j k}^{* i}$, though it is not in general metrical, even if the original connection is metrical.

\section{$\S 3$. The normal metric.}

$10^{\circ}$. Let $C$ be a curve in $\mathfrak{\mho}$ defined by $x^{i}=x^{i}(t)$, and denote by $A$ and $B$ the points of $C$ with the respective parametric value $t_{0}$ and $t_{1}$. The equations

$$
x^{i}=x^{i}(t)+\varepsilon v^{i}(t),
$$

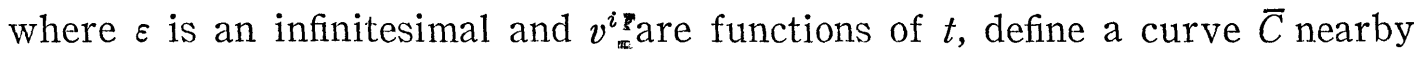
$C$ and passing through $A^{\prime}\left(t_{0}\right)$ and $B^{\prime}\left(t_{1}\right)$ which are nearby $A$ and $B$ respectively. Let a metric be introduced in $\mathfrak{F}$, and putting $F=\sqrt{g_{i j}\left(x, x^{\prime}\right) x^{i} x^{\prime j}}$, where $x^{i}=d x^{i} / d t$, the first variation of the absolute length of $C$ is given by

$$
\delta s=\varepsilon \int_{t_{0}}^{t_{1}}\left\{\frac{\partial F}{\partial x^{k}}-\frac{d}{d t}\left(\frac{\partial F}{\partial x^{\prime k}}\right)\right\} v^{k} d t+\left[\frac{\partial F}{\partial x^{\prime k}}\left(\varepsilon v^{k}\right)\right]_{t_{0}}^{t_{1}} .
$$


The curve satisfying the differential equaticns

$$
\frac{d}{d t}\left(\frac{\partial F}{\partial x^{\prime k}}\right)-\frac{\partial F}{\partial x^{k}}=0
$$

is called the stationary curve.

Now let $C$ be a stationary curve, and then we have

$$
\delta s=\left[\frac{\partial F}{\partial x^{\prime k}}\left(\varepsilon v^{k}\right)\right]_{t_{0}}^{t_{1}},
$$

where $\varepsilon v^{k}\left(t_{0}\right)$ and $\varepsilon v^{k}\left(t_{1}\right)$ are variations of coordinates of the extreme points $A$ and $B$ respectively. The direction of the vector $X$ satisfying

$$
\frac{\partial F}{\partial x^{\prime k}} X^{k}=0
$$

is called to be transversal to the stationary curve $C$, and if we translate the stationary curve $C$ such that its extreme points lie on the direction transversal to $C$, then the above variation $\delta s$ is zero.

Contrary to the case of Riemannian geometry, a transversality does not in general coincide with an orthogonality. In fact, we have

$$
\frac{\partial F}{\partial x^{\prime i}}=\frac{1}{F}\left(g_{i k} x^{\prime k}+\frac{1}{2} \frac{\partial g_{j k}}{\partial x^{\prime i}} x^{\prime j} x^{\prime k}\right),
$$

therefore $\partial F / \partial x^{\prime i} X^{i}=0$ does not in general give $g_{i k} x^{\prime k} X^{i}=0$. If we assume that the metric satisfies the equations

$$
\frac{\partial g_{i j}}{\partial y^{k}} y^{i}=0 \quad(j, k=1,2, \cdots, n),
$$

we obtain $\partial F / \partial x^{\prime i}=g_{i k} x^{\prime k} / F$, and consequently the transversality coincides with the orthogonality. Next, if in place of a general parameter $t$ we use the absolute arc-length $s$ of the curve, then the equations (15) are rewritten in the form

$$
\begin{aligned}
& \frac{d^{2} x^{i}}{d s^{2}}+\gamma_{j k}^{i} \frac{d x^{j}}{d s} \frac{d x^{k}}{d s} \\
& \quad+g^{i n} \frac{\partial g_{j h}}{\partial x^{\prime k}} \frac{d x^{j}}{d s}-\frac{d^{2} x^{k}}{d s^{2}}+\frac{1}{2} g^{i h} \frac{d}{d s}\left(\frac{\partial g_{j k}}{\partial x^{\prime h}} \frac{d x^{j}}{d s} \frac{d x^{k}}{d s}\right)=0
\end{aligned}
$$

which are the differential equations of the stationary curve, where

and

$$
\gamma_{j k}^{i}=g^{i h} \gamma_{j h k},
$$

$$
\gamma_{j h k}=\frac{1}{2}\left(\frac{\partial g_{j h}}{\partial x^{k}}+\frac{\partial g_{h k}}{\partial x^{j}}-\frac{\partial g_{k j}}{\partial x^{-}}\right) .
$$

If we assume that the metric satisfies (16), then we see immediately that (17) has the familiar form in Riemannian geometry, i.e. 


$$
\frac{d^{2} x^{i}}{d s^{2}}+\gamma_{j k}^{i} \frac{d x^{j}}{d s} \frac{d x^{k}}{d s}=0 .
$$

Since $\partial g_{i j} / \partial y^{k}$ are the components of the tensor field of type $(0,3)$, the condition (16) does not depend on the special coordinate system. Such a metric is called to be normal and from now on we shall consider the normal metric alone.

$11^{\circ}$. Let us find an example of a metrical connection in the space with the normal metric and from this determine the general form of connections which may be defined in our space. To do so it is sufficient to find $\Gamma_{j k}{ }^{i}$ and $C_{j k}{ }^{i}$ satisfying the three conditions of a connection. With the condition (8) in our mind we put

$$
C_{j h k}=\begin{array}{ll}
1 & \partial g_{j n} \\
2 & \partial y^{k}
\end{array}
$$

and then these $C_{j h k}$ are differentiable functions on $\mathfrak{B}(\mathfrak{F})$, which satisfy all of the necessary conditions for coefficients of a connection. Hence in order to solve (1), it is sufficient to find $\Gamma_{j n k}$ such that

$$
\Gamma_{\beta \lambda \gamma}=\Gamma_{j h k} \frac{\partial x^{j}}{\partial x^{\beta}} \frac{\partial x^{h}}{\partial x^{\lambda}} \frac{\partial x^{k}}{\partial x^{\gamma}}+g_{j h} \frac{\partial x^{h}}{\partial x^{\lambda}} \frac{\partial^{2} x^{j}}{\partial x^{\beta} \partial x^{\gamma}}+\frac{1}{2} \frac{\partial g_{j h}}{\partial y^{h}} \frac{\partial x^{j}}{\partial x^{\beta}} \frac{\partial x^{h}}{\partial x^{\lambda}} \frac{\partial^{2} x^{k}}{\partial x^{r} \partial x^{\delta}} \frac{\partial x^{\delta}}{\partial x^{l}} y^{l} .
$$

On the other hand $\gamma_{j n k}$ satisfy the following equations of transformation:

$$
\begin{gathered}
\gamma_{\beta \lambda \gamma}=\gamma_{j h k} \frac{\partial x^{j}}{\partial x^{\beta}} \frac{\partial x^{h}}{\partial x^{\lambda}} \frac{\partial x^{k}}{\partial x^{r}}+g_{j h} \frac{\partial x^{h}}{\partial x^{\lambda}} \frac{\partial^{2} x^{j}}{\partial x^{\beta} \partial x^{r}} \\
+\frac{1}{2} \frac{\partial g_{j h}}{\partial y^{k}}\left(\frac{\partial x^{j}}{\partial x^{\beta}} \frac{\partial x^{h}}{\partial x^{\lambda}} \frac{\partial^{2} x^{k}}{\partial x^{r} \partial x^{\delta}}+\frac{\partial x^{j}}{\partial x^{\lambda}} \frac{\partial x^{h}}{\partial x^{r}}-\frac{\partial^{2} x^{h}}{\partial x^{\beta} \partial x^{\delta}}-\frac{\partial x^{j}}{\partial x^{r}} \frac{\partial x^{h}}{\partial x^{\beta}{ }_{j}} \frac{\partial^{2} x^{k}}{\partial x^{\lambda} \partial x^{\delta}}\right) \frac{\partial x^{\delta}}{\partial x^{l}} y^{l} .
\end{gathered}
$$

Hence if we put $\mu_{j h k}=\Gamma_{j h k}-\gamma_{j h k}$, then we have

$$
\begin{aligned}
\mu_{\beta \lambda \gamma}= & \mu_{j h k} \frac{\partial x^{j}}{\partial x^{\beta}} \partial x^{h} \partial x^{\lambda} \partial x^{k} \\
& -\frac{1}{2} \frac{\partial g_{j h}}{\partial y^{k}}\left(\frac{\partial x^{j}}{\partial x^{\lambda}} \frac{\partial x^{h}}{\partial x^{\gamma}} \frac{\partial^{2} x^{k}}{\partial x^{\beta} \partial x^{\delta}}-\frac{\partial x^{j}}{\partial x^{r}} \frac{\partial x^{h}}{\partial x^{\beta}} \frac{\partial^{2} x^{k}}{\partial x^{\lambda} \partial x^{\delta}}\right) \frac{\partial x^{\delta}}{\partial x^{l}} y^{l} .
\end{aligned}
$$

Making use of (19) and the normality condition of the metric we have

$$
\gamma_{\beta}^{\mu} r y^{\beta} y r=\gamma_{j k}^{l} y^{j} y^{k} \frac{\partial x^{\mu}}{\partial x^{l}}+\frac{\partial^{2} x^{l}}{\partial x^{\beta} \partial x^{r}} \frac{\partial x^{\beta}}{\partial x^{b}} \frac{\partial x^{r}}{\partial x^{c}} y^{b} y^{c} \frac{\partial x^{\mu}}{\partial x^{l}} \text {. }
$$

Now we define

$$
G^{l}=\frac{1}{2} \gamma_{j k}^{l} y^{j} y^{k}
$$

and then a differentiation of $G^{\mu}$ by $y^{\lambda}$ gives

$$
\frac{\partial G^{\mu}}{\partial y^{\lambda}}=\frac{\partial G^{l}}{\partial y^{h}} \frac{\partial x^{\prime \prime}}{\partial x^{l}} \frac{\partial x^{h}}{\partial x^{\lambda}}+\frac{\partial^{2} x^{l}}{\partial x^{\beta} \partial x^{\lambda}} \frac{\partial x^{\beta}}{\partial x^{b}} \frac{\partial x^{\prime \prime}}{\partial x^{l}} y^{b} .
$$


Accordingly if we determine $\mu_{j h k}$ as

$$
\mu_{j h k}=\frac{1}{2}\left(\frac{\partial g_{j k}}{\partial y^{l}} \frac{\partial G^{l}}{\partial y^{n}}-\frac{\partial g_{h k}}{\partial y^{l}} \frac{\partial G^{l}}{\partial y^{j}}\right),
$$

then these satisfy $(20)$, and $r_{j h k}+\mu_{j h k}$ are differentiable functions on $\mathfrak{B}(\mathfrak{F})$ which are positively homogeneous of degree 0 . We state these results in the theorem:

THeOREm 1. In the space with the normal metric, the quantities

$$
\begin{aligned}
& \Gamma_{j h k}=\gamma_{j h k}+\mu_{j h k} \\
& C_{j h k}=\frac{1}{2} \frac{\partial g_{j h}}{\partial y^{k}}
\end{aligned}
$$

define a metrical connection. Moreover, its torsion tensor is zero and the absolute geodesic coincides with the stationary curve.

That the torsion tensor be zero is verified by proving

$$
r_{j h k} y^{j}=\frac{\partial G_{h}}{\partial y^{k}}
$$

Once we find one example of a connection, it is easy to determine the ; eneral form of the connection which may be defined in our space. We set

$$
\left\{\begin{array}{l}
\Gamma_{j h k}=\gamma_{j h k}+\mu_{j h k}+A_{j h k} \\
C_{j h k}=\frac{1}{2} \frac{\partial g_{j h}}{\partial y^{k}}+B_{j h k}
\end{array}\right.
$$

and consider the conditions which are satisfied by $A_{j h k}$ and $B_{j h k}$, and then we have easily the following results.

TheоReм 2. Let $A_{j h k}$ and $B_{j h k}$ be any differentiable functions on $\mathfrak{B}(\mathfrak{F})$ satisfying the following equations of transformation:

$$
\begin{aligned}
& A_{\beta \lambda \gamma}=A_{j h k} \frac{\partial x^{j}}{\partial x^{\beta}} \frac{\partial x^{h}}{\partial x^{\lambda}} \frac{\partial x^{k}}{\partial x^{r}}+B_{j h k} \frac{\partial x^{j}}{\partial x^{\beta}} \frac{\partial x^{h}}{\partial x^{\lambda}} \frac{\partial^{2} x^{k}}{\partial x^{\prime} \partial x^{\delta}} \frac{\partial x^{\delta}}{\partial x^{l}} y^{l} \\
& B_{\beta \lambda r}=B_{j h k} \frac{\partial x^{j}}{\partial x^{\beta}} \frac{\partial x^{h}}{\partial x^{\lambda}} \frac{\partial x^{k}}{\partial x^{r}}
\end{aligned}
$$

such that $A_{j h k}$ and $B_{j h k}$ are positively homogeneous of degree 0 and -1 respectively, and the latter yields the condition $B_{j n k} y^{k}=0$. Then the coefficients $\Gamma_{j n k}$ and $C_{j h k}$ of the general connection which may be defined in the space with the normal metric are given by (22).

Further, the condition that the connection be metrical is expressible in the terms that both of $A_{j h k}$ and $B_{j h k}$ be skew-symmetric with respect to the first two indices.

$12^{\circ}$. Through these considerations of the space with line-elements, we shall notice that it would be hardly expected that analogous properties still 
hold in our space as in a Riemannian space, unless we assume the metric to be normal. Actually the normal metric is nothing else but a Finsler metric. Thus we have a characterization of a Finsler space.

Theorem 3. A necessary and sufficient condition that a metric be normal, is that the metric be a Finsler metric.

Proof. Putting $F=\sqrt{g_{i j}(x, y) y^{i} y^{j}}$, we have

$$
\frac{1}{2} \frac{\partial^{2} F^{2}}{\partial y^{i} \partial y^{j}}=g_{i j}+\frac{\partial g_{m i}}{\partial y^{j}} y^{m}+\frac{1}{2} \frac{\partial}{\partial y^{j}}\left(\frac{\partial g_{m n}}{\partial y^{i}} y^{m} y^{n}\right)
$$

from which it follows $g_{i j}=\frac{1}{2}-\partial^{2} F^{2} / \partial y^{i} \partial y^{j}$ by the normality condition of the metric. Moreover, $F$ as above defined satisfies the conditions imposed upon the fundamental metric function in a Finsler space. Hence $g_{i j}$ define a Finsler metric. The sufficiency is immediate from the homogeneity of a Finsler metric.

\section{$\S 4$. The connection of $\mathbb{E}$. Cartan.}

$13^{\circ}$. We shall notice from the above discussions that the connection stated in Theorem 1 is the shortest and the fittest. Of course, if we put $C_{j h k}=0$, it might surely be the shortest, but it is not metrical by Lemma 1 . So it may be quite natural that we use $\partial g_{j h} / \partial y^{k}$ which are directly derived from the most fundamental $g_{i j}$. So in Theorem 2 we put $B_{j h k}=0$. (This condition is geometrically characterized as follows. Let us define the absolute differential $D X$ of a vector field $X$ on $\Im$ by

$$
D X^{i}=d X^{i}+\Gamma_{j k}{ }^{i} X^{j} d x^{k}+C_{j k}{ }^{i} X^{j} d y^{k}
$$

and denote by $\tilde{D} X$ the absolute differential for $d x^{i}=0(i=1, \cdots, n)$. If we assume $\tilde{g}[\tilde{D} X, Y]=\tilde{g}[X, \tilde{D} Y]$ for any vectors $X$ and $Y$ with a same origin, then we have $B_{j h k}=0$ (cf. [4]) ). Thus $A_{j h k}$ are the components of a tensor field. Hence the general form of $\Gamma_{j h k}$ is $\gamma_{j h k}+\mu_{j h k}$ added by a component of any tensor field of type $(0,3)$. And then if we assume that its torsion tensor be zero, we get $A_{j n k}=0$. This is the connection defined by E. Cartan [4], which has the desirable properties, as seen in Theorem 1. Thus we notice that the connection defined by $\mathrm{E}$. Cartan is the shortest and the fittest. Moreover, it is quite general in the sense that we can derive from it parallel displacements deñned by other authors. We shall see this in the following.

$14^{\circ}$. Let the connection of $\mathrm{E}$. Cartan be defined in the space $\mathfrak{F}$. In this case the equations (3) become as follows:

$$
\frac{d X^{i}}{d t}+\left(r_{j k}^{i}+\mu_{j k}^{i}\right) X^{j} \frac{d x^{k}}{d t}+\frac{1}{2} g^{i h} \frac{\partial g_{j h}}{\partial y^{k}} X^{j} \frac{d y^{k}}{d t}=0 .
$$


The parallel displacement of a vector along a curve $C$ with respect to $C_{a} \ldots$ the one defined by Synge [1] and Taylor [2]. In fact, since

$$
\mu_{j h k}\left(x, \frac{d x}{d t}\right) \frac{d x^{k}}{d t}=0,
$$

(24) is reduced to their form

$$
\frac{d X^{i}}{d t}+\gamma_{j k}^{i} X^{j} \frac{d x^{k}}{d t}+\frac{1}{2} g^{i n} \frac{\partial g_{j h}}{\partial y^{k}} X^{j} \frac{d y^{k}}{d t}=0,
$$

where $y^{i}=d x^{i} / d t$.

Since

$$
g_{i j}=\frac{1}{2} \frac{\partial^{2} F^{2}}{\partial y^{i} \partial y^{j}}
$$

where $F=\sqrt{g_{i j}(x, y) y^{i} y^{j}}$, we have

$$
\begin{aligned}
& 1 \\
& 2 \\
& 2
\end{aligned} \frac{g_{j h}}{\partial y^{k}}=\frac{1}{2}\left(\frac{\partial g_{j h}}{\partial y^{k}}+\frac{\partial g_{h k}}{\partial y^{j}}-\frac{\partial g_{k j}}{\partial y^{h}}\right),
$$

which have the similar form as Christoffel symbols. It is as a formal generalization of the parallelism in Riemannian geometry that Synge and Taylor defined the parallelism in $\widetilde{F}$ by the equations (25), However the quantities $\gamma_{j k}^{i}$ and $\frac{1}{2} g^{i n} \partial g_{j h} / \partial y^{k}$ do not satisfy the first condition of a connection and so have no geometrical meaning except absolutely parallel displacements.

And the parallel displacement of a vector $X$ with respect to $\tilde{C}_{X}$ is the one defined by Barthel [7], where $\widetilde{C}_{X}$ is the curve as stated in $2^{\circ}$ and $X$ is the vector itself to be displaced. In fact, in this case we have $\partial g_{j h}(x, X) /$ $\partial y^{k} X^{j}=0$ and since $G^{l}$ is positively homogeneous of degree 2, we have $\partial G^{l}(x, X) / \partial y^{j} X^{j}=2 G^{l}(x, X)$, whence it follows that

$$
\mu_{j h k}(x, X) X^{j}=-\frac{1}{2} \frac{\partial g_{h k}(x, X)}{\partial y^{l}} \gamma_{j m}^{l} X^{j} X^{m},
$$

so (24) is reduced to his form

$$
\frac{d X^{i}}{d t}+\left(\gamma_{j k}^{i}-\frac{1}{2} g^{i n} \frac{\partial g_{h k}}{\partial y^{l}} \gamma_{j m}^{l} X^{m}\right) X^{j} \frac{d x^{k}}{d t}=0,
$$

where $y^{i}=X^{i}$.

The quantities $B_{j k}{ }^{i}$ defined by

$$
B_{j k}{ }^{i}=\gamma_{j k}{ }^{i}-\frac{1}{2} g^{i n} \frac{\partial g_{h k}}{\partial y^{l}} \gamma_{j m}{ }^{l} y^{n}
$$

and $C_{j k}^{* i}(=0)$ do not also satisfy the first condition of a connection and so have no geometrical meaning except the parallel displacement with respect to the vector itself. But contrary to the case of Synge and Taylor we can define the absolute differential $D X$ of a vector field $X$ by

$$
D X^{i}=d X^{i}+B_{j k}^{i} X^{j} d x^{k},
$$


where $y^{i}=X^{i}$, though the operation $D$ is not linear. Since in (25) the term $d^{2} x^{i} / d t^{2}$ is contained, in the case of Synge and Taylor we can not define the absolute differential (23).

Barthel introduced the notion of the volume into Finsler spaces regarded as locally Minkowskian spaces and defined this connection, which has no ambiguity of the supporting element.

Thus the parallelisms of Synge-Taylor and Barthel are special cases of the metrical one of E. Cartan and so they are metrical.

$15^{\circ}$. We remarked in Lemma 2 that $\Gamma_{j k}^{* i}$ and $C_{j k}^{* i}(=0)$ define a new connection, if $C_{j k}^{i} y^{j}=0$ as the case of the connection of $\mathrm{E}$. Cartan. In this case we have

$$
\begin{aligned}
\Gamma_{j h k}^{*} & =\Gamma_{j h k}-C_{j h l} \Gamma_{m k}^{l} y^{m} \\
& =\gamma_{j h k}-\frac{1}{2}\left(\begin{array}{c}
\partial g_{j h} \\
\partial y^{l}
\end{array} \frac{\partial G^{l}}{\partial y^{k}}+\frac{\partial g_{h k}}{\partial y^{l}} \frac{\partial G^{l}}{\partial y^{j}}-\frac{\partial g_{j k}}{\partial y^{l}} \partial G^{l}\right.
\end{aligned}
$$

where $G^{l}=\frac{1}{2} \gamma_{m}{ }_{n} y^{m} y^{n}$. These are also expressed as follows:

$$
\begin{aligned}
& \Gamma_{j h k}^{*}=\gamma_{j h k}-\frac{1}{2}\left(\frac{\partial g_{j h}}{\partial y^{l}} \gamma_{m k}^{l}+\frac{\partial g_{h k}}{\partial y^{l}} \gamma_{m^{2}}^{l}-\frac{\partial g_{k j}}{\partial y^{l}} \gamma_{m h}^{l}\right) y^{y^{m}}
\end{aligned}
$$

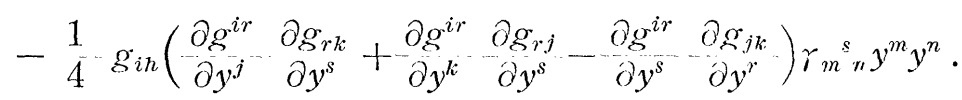

In fact, from the definition of $\gamma_{j h k}$, we have

$$
\frac{\partial r_{j h k}}{\partial y^{l}} y^{j} y^{k}=0
$$

hence

$$
\begin{aligned}
\frac{\partial G^{l}}{\partial y^{k}} & =\gamma_{m k}{ }_{k} y^{m}+\frac{1}{2} \frac{\partial \gamma_{m}{ }^{l}}{\partial y^{k}} y^{m} y^{n} \\
& =\gamma_{m}{ }_{k}{ }_{k} y^{m}+\frac{1}{2} \partial g^{l r} y^{l i} \gamma_{m r n} y^{m} y^{n} \\
& =\gamma_{m}{ }_{k}^{l} y^{m}+\frac{1}{2} g_{r s} \frac{\partial g^{l r}}{\partial y^{k}} \gamma_{m}{ }_{n}{ }_{n} y^{m} y^{n} \\
& =\gamma_{m}{ }_{k}^{l} y^{m}-\frac{1}{2} g^{l r} \frac{\partial g_{r s}}{\partial y^{k}} \gamma_{m}{ }_{n}^{s} y^{m} y^{n} .
\end{aligned}
$$

Since we see by (26) that

we have

$$
\frac{\partial g_{r s}}{\partial y^{k}}=\frac{\partial g_{r k}}{\partial y^{s}}, \frac{\partial g_{j h}}{\partial y^{l}}=\frac{\partial g_{h l}}{\partial y^{j}},
$$

$$
\begin{aligned}
\frac{\partial g_{j h}}{\partial y^{l}} \frac{\partial G^{l}}{\partial y^{k}} & =\frac{\partial g_{j h}}{\partial y^{l}} \gamma_{m}{ }^{l} y^{m}-\frac{1}{2} g^{l r} \frac{\partial g_{r k}}{\partial y^{s}} \frac{\partial g_{h l}}{\partial y^{j}} \gamma_{m}{ }_{n}{ }_{n} y^{m} y^{n} \\
& =\frac{\partial g_{j h}}{\partial y^{l}} \gamma_{m}{ }_{k} y^{m}+\frac{1}{2} g_{h l} \frac{\partial g^{l r}}{\partial y^{j}} \partial g_{r k} \quad y^{s} \gamma_{m}{ }_{n}{ }_{n} y^{m} y^{n},
\end{aligned}
$$


and if we substitute these into (29), then we obtain (30). These are exactly the coefficients $P_{j h k}^{*}$ of the connection defined by Rund [6].

When this connection is defined, the parallel displacement of a vector along a curve $C$ with respect to $\widetilde{C}_{a}$ is the one defined by $P_{j h k}$ of Rund [5], that is:

where

$$
\frac{d X^{i}}{d t}+P_{j k}^{i} X^{j} \frac{d x^{k}}{d t}=0
$$

$$
P_{j k}^{i}=\gamma_{j k}^{i}-1-g^{i h} \frac{\partial g_{j h}}{\partial y^{l}-\gamma_{m k}{ }^{l} y^{m}}
$$

and $y^{i}=a(t) d x^{i} / d t$.

Rund defined geometrically the covariant derivatives in his paper [5] from the point of view that one should regard Finsler spaces as locally Minkowskian spaces. In his paper [6], in which he investigated the curvature properties from analytical aspects, he generalized for this purpose the coefficients $P_{j k}^{i}$ in [5] to $P_{j k \text {. }}^{* i}$.

And in the case of the connection by $P_{j k}^{* i}$ the parallel displacement with respect to $\widetilde{C}_{X}$ is again Barthel's. Though the connection defined by Rund is not in general metrical, this is metrical as stated in $14^{\circ}$.

$16^{\circ}$. Finally we shall apply Lemma 3 to the connection of E. Cartan. Since $\Gamma_{h}{ }_{l}^{i} y^{h} y^{l}=\gamma_{h}{ }_{l}^{i} y^{h} y^{l}$, we obtain immediately

$$
\begin{aligned}
\tilde{\Gamma}_{j k}{ }^{i} & =\frac{1}{2} \frac{\partial^{2}}{\partial y^{j} \partial y^{k}}\left(\gamma_{h}{ }^{i} y^{\left.{ }^{h} y^{l}\right)}\right. \\
& =\gamma_{j k}{ }^{i}+\left(\frac{\partial \gamma_{h}{ }^{i}{ }_{j}}{\partial y^{k}}+\frac{\partial \gamma_{h}{ }^{i}{ }_{k}}{\partial y^{j}}\right) y^{h}+\frac{1}{2} \frac{\partial^{2} \gamma_{h}{ }^{i}{ }_{l}}{\partial y^{j} \partial y^{k}} y^{h} y^{l},
\end{aligned}
$$

which are the coefficients of the connection defined by Berwald [3] making use of the differential equations of the paths.

Also, in case a connection be defined by $\tilde{\Gamma}_{j k}^{i}$ and $C_{j k}^{* i}(=0)$, it is casily verified that the parallelisms with respect to $\widetilde{C}_{a}$ and $\tilde{C}_{X}$ coincide with the one defined by $P_{j k}^{i}$ of Rund and with the one of Barthel respectively.

Thus we have proved

Theorem 4. Let the connection of E. Cartan be defined in a Finsler space F. The parallel displacement of a vector with respect to $\widetilde{C}_{a}$ is the one defined by Synge and Taylor, and the one with respect to the vector itself is the one defined by Barthel.

The connections defined by $P_{j k}^{* i}$ of Rund and by Berwald are obtained by applying Lemma 2 and Lemma 3 respectively to Cartan's connection. In both cases the parallel displacement of a vector with respect to $\tilde{C}_{a}$ is the one defined by $P_{j k}^{i}$ of Rund, and the one with respect to the vector itself is again Barthel's.

Mathematical Institute, University of Kyoto. 


\section{References}

[1] J.L. Synge, A generalization of the Riemannian line-element, Trans. Amer. Math. Soc., 27 (1925), 61-67.

[2] J.H. Taylor, A generalization of Levi-Civita's parallelism and the Frenet formulas, Trans. Amer. Math. Soc., 27 (1925), 246-264.

[3] L. Berwald, Über Parallelübertragung in Räumen mit allgemeiner Massbestimmung, Jber. Deutsch. Math. Verein., 34 (1926), 213-220.

[4] E. Cartan, Les espaces de Finsler, Paris. 1934.

[5] H. Rund, Über die Parallelverschiebung in Finslerschen Räume, Math. Z., 54 (1951), 115-128.

[6] H. Rund, On the analytical properties of curvature tensors in Finsler spaces, Math. Ann., 127 (1954) 82-104.

[7] W. Barthel, Über eine Parallelverschiebung mit Längeninvarianz in lokalMinkowskischen Räumen, Arch. Math., 4 (1953), 346-365. 